\title{
High-fat Diet Increases Phospholipid Peroxidation in the Liver of Mature Fischer 344 Rats
}

\author{
Saki Hayasaka ${ }^{1}$, Fumiko Kimura², Shunji Kato ${ }^{1}$, Naoki Shimizu', Junya Ito', \\ Oki Higuchi $^{3}$, Katsuhiro Izumisawa ${ }^{4}$, Teruo Miyazawa ${ }^{1,5}$ and Kiyotaka Nakagawa ${ }^{1, *}$ \\ ${ }^{1}$ Food and Biodynamic Chemistry Laboratory, Graduate School of Agricultural Sciences, Tohoku University, Sendai, JAPAN \\ ${ }^{2}$ Department of Human Health and Nutrition, Shokei Gakuin University, Natori, JAPAN \\ ${ }^{3}$ Biodynamic Plant Institute CO., Ltd., Sapporo, JAPAN \\ ${ }^{4}$ Eisai CO., Ltd., Tokyo, JAPAN \\ ${ }^{5}$ Food Biotechnology Innovation Project, NICHe, Tohoku University, Sendai, JAPAN
}

\begin{abstract}
Phospholipid peroxidation is considered to be involved in the pathophysiology of various diseases. While dietary antioxidants are believed to help prevent these diseases via inhibition of phospholipid peroxidation, further evaluation is needed to prove this hypothesis. For this, it is crucial to establish an animal model with accelerated phospholipid peroxidation. In this study, we hypothesized that a combination of aging and high-fat diet feeding may accelerate phospholipid peroxidation in vivo. High-fat diets were fed to mature and juvenile Fischer 344 rats for 12 weeks. The mature rats in particular accumulated body fat and liver phosphatidylcholine hydroperoxide ( $\mathrm{PCOOH})$. Interestingly, the increase in $\mathrm{PCOOH}$ levels was abrogated by the co-administration of antioxidants to mature rats. This may be attributed to factors including the decrease in body fat, functions of vitamin $E$, and/or the involvement of antioxidant-related genes, each caused by antioxidant administration. These results indicate that the high-fat diet-fed aging animal model may be suitable for investigation of the relationship between phospholipid peroxidation, oxidative stress-related diseases, and dietary antioxidants.
\end{abstract}

Key words: antioxidant, liver, oxidative stress, phosphatidylcholine hydroperoxide, rat

\section{INTRODUCTION}

Phospholipid peroxidation is considered to be involved in the pathophysiology of human diseases such as arteriosclerosis, diabetes, and dementia ${ }^{1-5)}$. While dietary antioxidants (e.g., vitamin $\mathrm{E}$, carotenoids, vitamin $\mathrm{C}$ and polyphenols) are believed to help prevent these diseases via inhibition of phospholipid peroxidation ${ }^{6-8)}$, further evaluation is needed to prove this oxidation and antioxidation hypothesis. To this end, the establishment of an animal model with accelerated phospholipid peroxidation will be highly valuable.

With the exception of administering certain chemicals such as carbon tetrachloride ${ }^{7,9)}$, aging and high-fat diet feeding are the most plausible ways to accelerate phospholipid peroxidation in vivo. Studies indicate that phospholipid peroxidation[e.g., phosphatidylcholine hydroperoxide (PCOOH) formation] increases in the liver of mature rats ${ }^{10)}$, and in rats receiving high-fat diet ${ }^{8)}$. However, other studies also suggest that the individual effects of aging or high fat diet feeding are limited and/or occasionally vary among animals, and that it takes a significant amount of time(e.g., over 7-18 month $^{10)}$ ) to amplify oxidative stress. We therefore hypothesized that the combination of aging and highfat diet feeding may provide a promising way to elevate and accelerate phospholipid peroxidation in vivo. To the best of our knowledge, very few studies have examined the effect of the combination of aging and high-fat diet feeding on $\mathrm{PCOOH}$ formation.

In this study, to evaluate this hypothesis, we fed high-fat diets to mature (20 weeks old) and juvenile (control; 6 weeks old) Fischer 344 rats. As expected, liver PCOOH levels increased significantly after 12 weeks of high-fat diet feeding. Since we employed Fischer 344 rats (known to rapidly accumulate body fat vis-à-vis other rat strains ${ }^{11)}$ ) and high-linoleic safflower oil[enhances the levels of easily oxidizable fatty acids (polyunsaturated fatty acids) in tissues $\left.^{12)}\right]$, therefore, we speculated that these factors, along with aging and high-fat diet feeding, may further ac-

\footnotetext{
*Correspondence to: Kiyotaka Nakagawa, Food and Biodynamic Chemistry Laboratory, Graduate School of Agricultural Sciences, Tohoku University, Sendai, JAPAN

E-mail: nkgw@biochem.tohoku.ac.jp

Accepted January 29, 2017 (received for review December 5, 2016)

Journal of Oleo Science ISSN 1345-8957 print / ISSN 1347-3352 online

http://www.jstage.jst.go.jp/browse/jos/ http://mc.manusriptcentral.com/jjocs
} 
celerate $\mathrm{PCOOH}$ formation. Furthermore, the increase in $\mathrm{PCOOH}$ levels was abrogated by the co-administration of dietary antioxidants. Therefore, such an animal model with high tissue $\mathrm{PCOOH}$ levels will become valuable to study the relationship between phospholipid peroxidation, oxidative stress-related diseases, and dietary antioxidants.

\section{EXPERIMENTAL}

\subsection{Animals and diets}

Male 6 weeks old Fisher 344/Jcl rats, and 20 weeks old Fisher 344, retired male breeders were obtained from CLEA Japan, Inc. (Tokyo, Japan). In this study, we defined 6 weeks old rats as juvenile (control) and 20 weeks old rats as mature. Rats were housed individually in stainless steel wire mesh cages at a constant room temperature of $24 \pm$ $1^{\circ} \mathrm{C}$ with a $12 \mathrm{~h}$ light-dark cycle (light period: 8:00-20:00) and acclimated to the animal housing with ad libitum commercial laboratory chow (CE-2; CLEA Japan Inc.) .

After 1 week of acclimation, mature $(n=5)$ and juvenile $(n=8)$ rats were fed with $20 \mathrm{wt} \%$ high-fat diet (modified AIN-93G diet supplemented with high-linoleic safflower oil, Table 1) for 12 weeks. Mature $(n=5)$ and juvenile $(n=8)$ rats were also fed with $20 \mathrm{wt} \%$ high-fat diet supplemented with $1 \mathrm{wt} \%$ commercial dietary antioxidant mix $(0.14 \mathrm{wt} \%$ vitamin $\mathrm{E}, 0.67 \mathrm{wt} \%$ fish oil, $0.018 \mathrm{wt} \%$ tomato polyphenol, 0.018 wt\% green tea polyphenol, 0.018 wt\% grape polyphenol, 0.018 wt\% cacao polyphenol, 0.018 wt\% blueberry polyphenol, 0.037 wt\% black soybean polyphenol; Yubera zeitaku polyphenol Kiwami from Eisai Co., Tokyo,

Table 1 Dietary compositions of the experimental diets.

\begin{tabular}{lcc}
\hline & HFD & HFD+Antiox \\
\hline Casein & 20.00 & 20.00 \\
L-Cystine & 0.30 & 0.30 \\
Cornstarch & 26.75 & 25.75 \\
Q-Cornstarch & 13.20 & 13.20 \\
Sucrose & 10.00 & 10.00 \\
High-linoleic safflower oil & 20.00 & 20.00 \\
Cellulose & 5.00 & 5.00 \\
AIN-93G mineral mix & 3.50 & 3.50 \\
AIN-93 vitamin mix & 1.00 & 1.00 \\
Choline bitartrate & 0.25 & 0.25 \\
t-Butylhydroquinone & 0.0014 & 0.0014 \\
Antioxidant mix* & - & 1.00 \\
\hline & & (g/100 g diet) \\
* Compositional details of the commercial dietary antioxidant \\
mix are described in the main article.
\end{tabular}

Japan). The major vitamin E components were 90.7 wt $\%$ $\alpha$-tocopherol(Toc), 4.4 wt $\% \gamma$-tocotrienol(T3), and 3.4 wt $\% \alpha$-T3. Fish oil included 28 wt $\%$ eicosapentaenoic acid (EPA) and 14 wt\% docosahexaenoic acid(DHA). During the experimental period, rats had free access to the experimental diet and distilled water. Body weights and food consumption were measured every other day. The experiments were conducted according to Regulations for Animal Experiments and Related Activities at Tohoku University and the protocol was approved by the institutional Animal Care and Use Committee of Tohoku University (2013-AgA009).

\subsection{Sampling of liver, plasma, and other tissues}

After feeding experimental diets, the rats were fasted for $12 \mathrm{~h}$ and euthanized by decapitation under isoflurane inhalation anesthesia. Tissues including liver and fat were collected and weighed. A small part of the liver ( 100 mg) was immediately immersed in RNAlater ${ }^{\circledR}$ solution(Life Technologies, Carlsbad, CA, USA) overnight, and was subsequently stored at $-30^{\circ} \mathrm{C}$ until PCR analysis. The remaining liver for biochemical experiments was perfused with cold saline, snap frozen in liquid $\mathrm{N}_{2}$, and stored at $-80^{\circ} \mathrm{C}$ until analysis. Blood was collected in heparinized tubes, and plasma was obtained from the blood by centrifugation $\left(4^{\circ} \mathrm{C}, 1000 \mathrm{~g}, 20 \mathrm{~min}\right)$ and stored at $-80^{\circ} \mathrm{C}$.

\subsection{Liver $\mathrm{PCOOH}$ analysis}

Liver $\mathrm{PCOOH}$ was measured by chemiluminescence detection-high-performance liquid chromatography (CLHPLC ${ }^{13)}$. Briefly, liver total lipids (TL) were extracted from liver homogenate by the Folch method ${ }^{14)}$, with $0.002 \%$ butylated hydroxytoluene (BHT). Liver TL were applied on a Sep-Pak Vac, 100 mg 1 cc $\mathrm{NH}_{2}$ cartridge (Waters Corporation, MA, USA) ${ }^{15)}$. The phospholipid (PL) fraction was eluted, and then subjected to CL-HPLC equipped with a Finepak SIL-NH ${ }_{2}-5$ column $(4.6 \times 250$ mm; JASCO Corporation, Tokyo, Japan) and a chemiluminescence detector (CLA-FL2; Tohoku Electronic Industries; Miyagi, Japan). The mobile phase was 2-propanol/methanol/water (135:45:20) at a flow rate of $1.0 \mathrm{~mL} / \mathrm{min}$. The post column chemiluminescence reagent for the specific detection of lipid hydroperoxides was a mixture of luminol and cytochrome $c$ in $50 \mathrm{mM}$ borate buffer $(\mathrm{pH} 10.0)$ at a flow rate of $1.5 \mathrm{~mL} / \mathrm{min}$. The concentration of $\mathrm{PCOOH}$ was calculated using an external standard prepared by a method previously reported by us ${ }^{15,16)}$.

\subsection{Measurement of liver TBARS, vitamin E, and other parameters}

Liver thiobarbituric acid reactive substances (TBARS) were obtained from TL via reaction with thiobarbituric acid in the presence of BHT and sodium dodecyl sulfate. The TBARS contents were determined by HPLC-fluorescence 
detection $^{17)}$. Liver vitamin $\mathrm{E}$ homologues, namely, $\alpha_{-}^{-}, \beta-, \gamma_{-}^{-}$, and $\delta$-Toc and $\alpha-, \beta-, \gamma-$, and $\delta$-T3, were determined by HPLC-MS/MS ${ }^{18)}$. Liver PL was determined as phosphorus in the TL by the modified Bartlett method ${ }^{19)}$. Triglyceride (TG) and total cholesterol (T-Cho) were measured using commercially available kits (Wako, Osaka, Japan). Plasma TL, TG, T-Cho, PL and nonesterified fatty acid (NEFA) were measured by the LSI Medience Corporation(Tokyo, Japan).

\subsection{Analysis of mRNA levels}

The mRNA levels of genes involved in the antioxidant system[Glutathione peroxidase1 (Gpx1), Glutathione peroxidase2 (Gpx2), catalase (Cat), Mn-Superoxide dismutase (Mn-Sod) and Cu/Zn-Superoxide dismutase (Cu/ $Z n$-Sod) ] were analyzed by real-time quantitative RT-PCR. Total mRNA was extracted from liver with a commercially available kit (Qiagen, Hilden, Germany) and converted to cDNA by Ready-To-Go ${ }^{\text {TM }}$ T-Primed First-Standard Kit (GE Healthcare, Piscataway, NJ, USA). Real-time PCR was performed on the CFX96 Real-Time PCR Detection System (Bio-Rad Laboratories, CA, USA) and SYBR Premix Ex Taq II(Takara, Shiga, Japan). All primer sequences used in this study have previously been reported ${ }^{8,20-23)}$.

\subsection{Statistical analysis}

Results are expressed as mean \pm standard deviation (SD). All statistical processing of the data was carried out by two-way factorial (diet $\times$ age) analysis of variance (twoway ANOVA). For factors where interaction was confirmed by two-way ANOVA, the Bonferroni test was further performed for multiple comparison. $P<0.05$ was considered statistically significant. All statistical analyses were performed using IBM SPSS Statistics for Windows, Version 22.0 (IBM Corp. in Armonk, NY).

\section{RESULTS AND DISCUSSION}

3.1 Food consumption, body weight, and tissue profile

As described in the Introduction, aging and high-fat diet feeding are two individual factors known to accelerate phospholipid peroxidation in vivo ${ }^{8,10)}$. However, their effect may be limited and takes a long time for oxidative stress to accumulate ${ }^{8,10)}$. In this study, we fed high-fat diets to mature (20 weeks old) and juvenile (control; 6 weeks old) Fischer 344 rats to evaluate whether the combination of aging and high-fat diet feeding will elevate as well as accelerate phospholipid peroxidation in vivo.

After 12 weeks of high-fat diet feeding to mature and juvenile rats, no difference was found in total food consumption between the two groups (Table 2), thus food efficiency was lower in mature rats than in juvenile rats. Mature rats that received high-fat diet showed greater body, liver, and mesenteric fat weights than juvenile rats. Mature rats also demonstrated higher concentrations of lipids (TL, TG and T-Cho) in the liver but not in plasma (Table 3).

Fischer 344 rats, compared with SD rats, are known to have lower food consumption and body weights but higher body fat percentage, and this tendency can be increased by feeding high-fat diets ${ }^{11)}$. Previous studies indicate that high-fat diet feeding increases body weight ${ }^{24,25)}$, body fat weight $^{8,25,26)}$, and liver lipids levels ${ }^{8,27)}$ compared to control diet feeding in Fischer 344 rats. McDonald et al. fed highfat diets to 5 months old and 2 years old Fischer 344 rats, and reported higher fat accumulation in older rats ${ }^{25)}$. These previous findings $\mathrm{s}^{8,11,24-27)}$, along with our results in the present study (Tables 2 and 3), indicate that the combination of aging and high-fat diet feeding can indeed enhance obesity of Fischer 344 rats in a rather short time.

Table 2 Body weights, total caloric food consumptions, and tissue weights in rats fed with high fat diets (HFD) or HFD supplemented with an antioxidant mix (HFD+Antiox) for 12 weeks.

\begin{tabular}{|c|c|c|c|c|c|c|c|}
\hline & \multicolumn{2}{|c|}{ Juvenile } & \multicolumn{2}{|c|}{ Mature } & \multirow{2}{*}{ age } & \multirow{2}{*}{ diet } & \multirow{2}{*}{ age $\times$ diet } \\
\hline & HFD & HFD+Antiox & HFD & HFD+Antiox & & & \\
\hline Initial body weight (g) & $140 \pm 5$ & $141 \pm 4$ & $323 \pm 3$ & $328 \pm 25$ & $* * *$ & ns & ns \\
\hline Final body weight (g) & $358 \pm 20$ & $349 \pm 26$ & $394 \pm 10$ & $401 \pm 19$ & $* * *$ & ns & ns \\
\hline Body weight increase (g) & $218 \pm 19$ & $207 \pm 25$ & $70.6 \pm 12.2$ & $73.0 \pm 8.4$ & $* * *$ & ns & ns \\
\hline Total food consumption (g) & $1096 \pm 48$ & $1037 \pm 72$ & $1082 \pm 27$ & $1090 \pm 241$ & ns & ns & ns \\
\hline Feed efficiency (g/100 kcal) & $4.31 \pm 0.20$ & $4.32 \pm 0.24$ & $1.41 \pm 0.22$ & $1.45 \pm 0.17$ & $* * *$ & ns & ns \\
\hline Liver (mg/g body weight) & $23.7 \pm 0.9$ & $24.4 \pm 1.4$ & $23.9 \pm 0.6$ & $26.0 \pm 0.6$ & $*$ & $* *$ & ns \\
\hline Epididymal fat (mg/g body weight) & $37.3 \pm 3.1$ & $35.2 \pm 3.5$ & $33.9 \pm 3.3$ & $34.3 \pm 2.2$ & ns & $* * *$ & ns \\
\hline $\begin{array}{l}\text { Perirenal-retroperitoneal fat } \\
\text { (mg/g body weight) }\end{array}$ & $38.6 \pm 1.7$ & $34.5 \pm 1.4$ & $37.7 \pm 2.5$ & $33.4 \pm 2.7$ & $\mathrm{~ns}$ & $* * *$ & ns \\
\hline Mesenteric fat (mg/g body weight) & $26.2 \pm 2.5$ & $23.6 \pm 3.2$ & $30.9 \pm 4.7$ & $28.8 \pm 3.2$ & $* * *$ & ns & ns \\
\hline
\end{tabular}

Values are expressed as mean $\pm \mathrm{SD}$ (juvenile; $\mathrm{n}=8$, mature; $\mathrm{n}=5$ ), * : $p<0.05, * *: p<0.01, * * *: p<0.001$ 
Table 3 Plasma and liver lipid contents of rats fed with high fat diets (HFD) or HFD supplemented with an antioxidant mix (HFD+Antiox) for 12 weeks.

\begin{tabular}{|c|c|c|c|c|c|c|c|}
\hline & \multicolumn{2}{|c|}{ Juvenile } & \multicolumn{2}{|c|}{ Mature } & \multirow{2}{*}{ age } & \multirow{2}{*}{ diet } & \multirow{2}{*}{ age $\times$ diet } \\
\hline & HFD & HFD+Antiox & HFD & HFD+Antiox & & & \\
\hline \multicolumn{8}{|c|}{ Plasma } \\
\hline $\mathrm{TL}(\mathrm{mg} / \mathrm{dL})$ & $488 \pm 111$ & $366 \pm 37$ & $467 \pm 38$ & $410 \pm 41$ & ns & $* * *$ & ns \\
\hline $\mathrm{TG}(\mathrm{mg} / \mathrm{dL})$ & $185 \pm 65$ & $120 \pm 19$ & $183 \pm 23$ & $154 \pm 24$ & ns & $* *$ & ns \\
\hline T-Cho (mg/dL) & $96 \pm 15$ & $80 \pm 7$ & $92 \pm 8$ & $84 \pm 5$ & ns & $* *$ & ns \\
\hline $\mathrm{PL}(\mathrm{mg} / \mathrm{dL})$ & $159 \pm 26$ & $126 \pm 13$ & $147 \pm 10$ & $130 \pm 10$ & ns & $* *$ & ns \\
\hline NEFA (mEq/L) & $1.13 \pm 0.32$ & $1.03 \pm 0.18$ & $1.14 \pm 0.10$ & $0.85 \pm 0.13$ & $\mathrm{~ns}$ & $*$ & ns \\
\hline \multicolumn{8}{|c|}{ Liver } \\
\hline $\mathrm{TL}(\mathrm{mg} / \mathrm{g})$ & $63.9 \pm 8.2$ & $91.8 \pm 22.3$ & $88.7 \pm 13.4$ & $127.4 \pm 15.4$ & $* * *$ & $* * *$ & ns \\
\hline TG (mg/g) & $22.6 \pm 2.7$ & $52.8 \pm 19.6$ & $49.6 \pm 16.1$ & $85.5 \pm 17.4$ & $* * *$ & $* * *$ & ns \\
\hline $\mathrm{TC}(\mathrm{mg} / \mathrm{g})$ & $2.12 \pm 0.35$ & $4.23 \pm 2.02$ & $4.44 \pm 2.05$ & $7.22 \pm 1.27$ & $* * *$ & $* * *$ & $\mathrm{~ns}$ \\
\hline PL (mg/g) & $29.1 \pm 6.3$ & $30.7 \pm 6.1$ & $34.7 \pm 10.6$ & $32.7 \pm 7.2$ & $\mathrm{~ns}$ & ns & ns \\
\hline
\end{tabular}

Values are expressed as mean $\pm \mathrm{SD}$ (juvenile; $\mathrm{n}=8$, mature; $\mathrm{n}=5$ ), * : $p<0.05,{ }^{* *}: p<0.01,{ }^{* * *}: p<0.001$

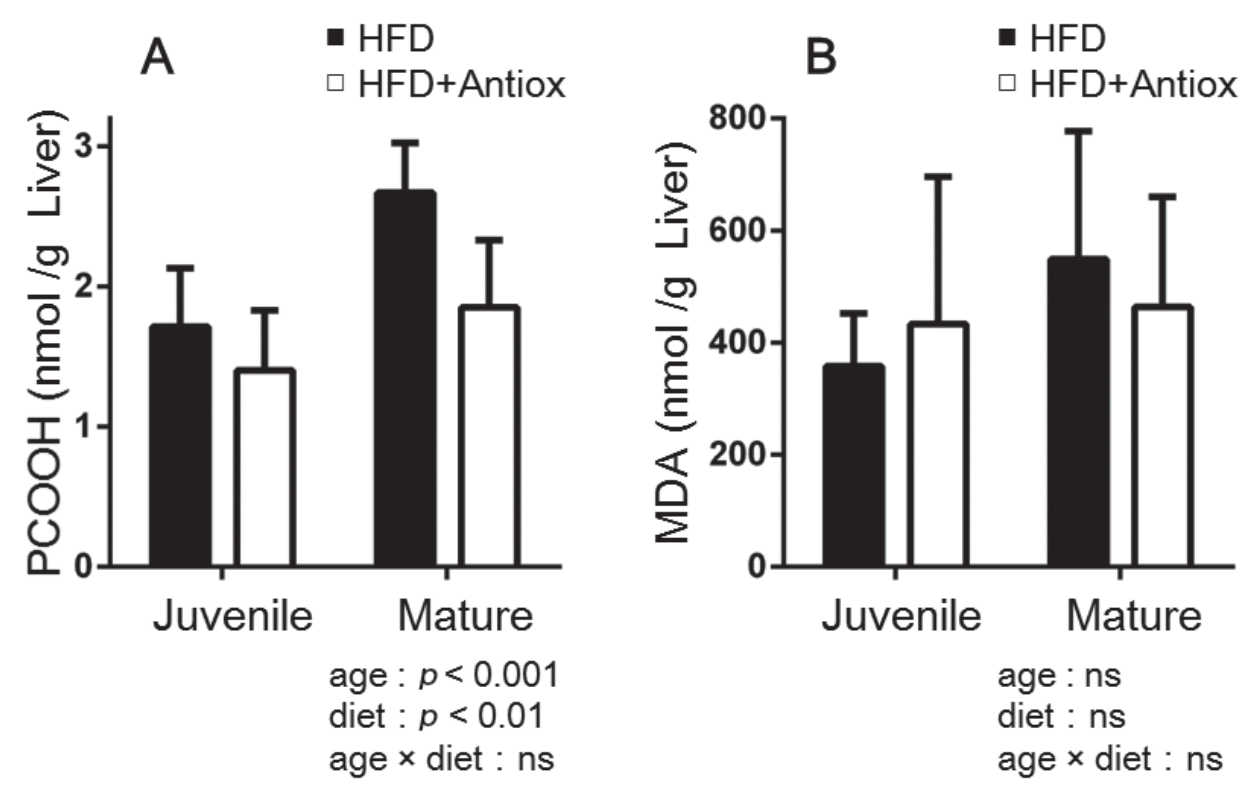

Fig. 1 Liver PCOOH(A) and TBARS(B) of mature or juvenile rats fed with experimental diets for 12 weeks. TBARS was expressed as MDA contents. Closed square; high fat diet(HFD), Open square; high fat diet supplemented with antioxidant mix (HFD + Antiox). Data are shown as means \pm SD, Juvenile $(n=8)$, Mature $(n=5)$.

\subsection{Liver phospholipid peroxidation and antioxidant pa- rameters}

As it was confirmed that obesity was enhanced in a short time with the combination of aging and high-fat diet feeding (Tables 2 and 3 ), we next determined whether peroxidation of phospholipids was accelerated in the Fischer 344 rats. To this end, $\mathrm{PCOOH}$ was measured as a reliable phospholipid peroxidation marker ${ }^{28,29)}$. We found that after 12 weeks of high-fat diet feeding, mature rats demonstrated higher PCOOH levels in the liver (Fig. 1), and thereby, it was confirmed that phospholipid peroxida- tion can be accelerated under the conditions used in this study, even without the administration of chemicals such as carbon tetrachloride. The $\mathrm{PCOOH}$ level in mature rats was approximately one and a half times higher than that in the juvenile group; this increase in $\mathrm{PCOOH}$ was almost equivalent to that found in the plasma $\mathrm{PCOOH}$ of hyperlipidemic patients ${ }^{2}$ and diabetic patients ${ }^{5}$.

Additionally, to identify why phospholipid peroxidation was accelerated in the liver, we examined the correlation between $\mathrm{PCOOH}$ and various parameters. We found a significant correlation between mesenteric fat weight and 


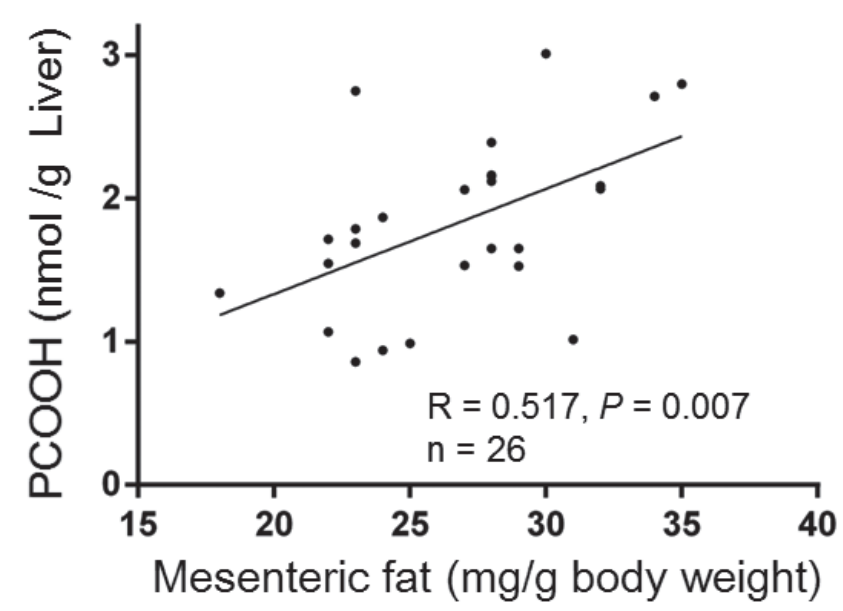

Fig. 2 The correlation between liver $\mathrm{PCOOH}$ and mesenteric fat. Data are shown as means $\pm \mathrm{SD}$, Juvenile $(n=8)$, Mature $(n=5)$.

$\operatorname{PCOOH}(\mathrm{R}=0.517, p=0.007)$ (Fig. 2). Compared with other fat tissues, mesenteric fat is more likely to develop inflammation $^{30)}$. Therefore, the accumulation of mesenteric fat that was observed in this study may have induced oxidative stress from inflammation (e.g., generation of inflammatory reactive oxygen species such as singlet oxygen), leading to the increase in liver $\mathrm{PCOOH}$ levels. It should also be noted that high-linoleic safflower oil, which is known to increase the levels of polyunsaturated fatty acids in tissues $^{12)}$, was used as the dietary fat source in this study. Further study is necessary to evaluate the effect of dietary fat source (high-linoleic safflower oil) on $\mathrm{PCOOH}$ accumulation in vivo.

In contrast to that of PCOOH, levels of TBARS (a classi- cal lipid peroxidation marker ${ }^{31)}$ ) did not significantly differ between mature and juvenile rat groups (Fig. 1). This contradiction may be attributed to the difference in the substances that were measured. The TBARS method is based on the measurement of malondialdehyde (MDA), which forms as a result of the degradation of lipid hydroperoxides. TBA reacts with MDA along with other various carbonyl compounds, resulting in a rather low specificity ${ }^{32}$. On the other hand, as the CL-HPLC method directly detects $\mathrm{PCOOH}^{28,29)}$, the method is more sensitive and suitable to determine the levels of liver phospholipid peroxidation. With regard to liver antioxidant (vitamin E) levels (Fig. 3) and mRNA levels of crucial antioxidant-related genes (Gpx1, Gpx2, Cat, Mn-Sod and Cu/Zn-Sod), these also did not differ between mature and juvenile rat groups (Table 4).

Based on these results, it was concluded that the combination of aging and high-fat diet feeding certainly increased $\mathrm{PCOOH}$ levels in the liver of mature Fischer 344 rats in a short period of time. The increase in liver $\mathrm{PCOOH}$ levels is presumably due to the mesenteric fat-induced oxidative stress from inflammation, rather than changes in the antioxidative factors (vitamin $\mathrm{E}$ and antioxidant-related genes) in the liver.

\subsection{Co-administration of dietary antioxidants}

The results mentioned above confirm, at least in part, that we were able to establish an animal model with accelerated phospholipid peroxidation. To validate its usability, we investigated the effect of antioxidant administration in the model. Co-administration of dietary antioxidants ameliorated PCOOH levels, but not those of TBARS in the liver
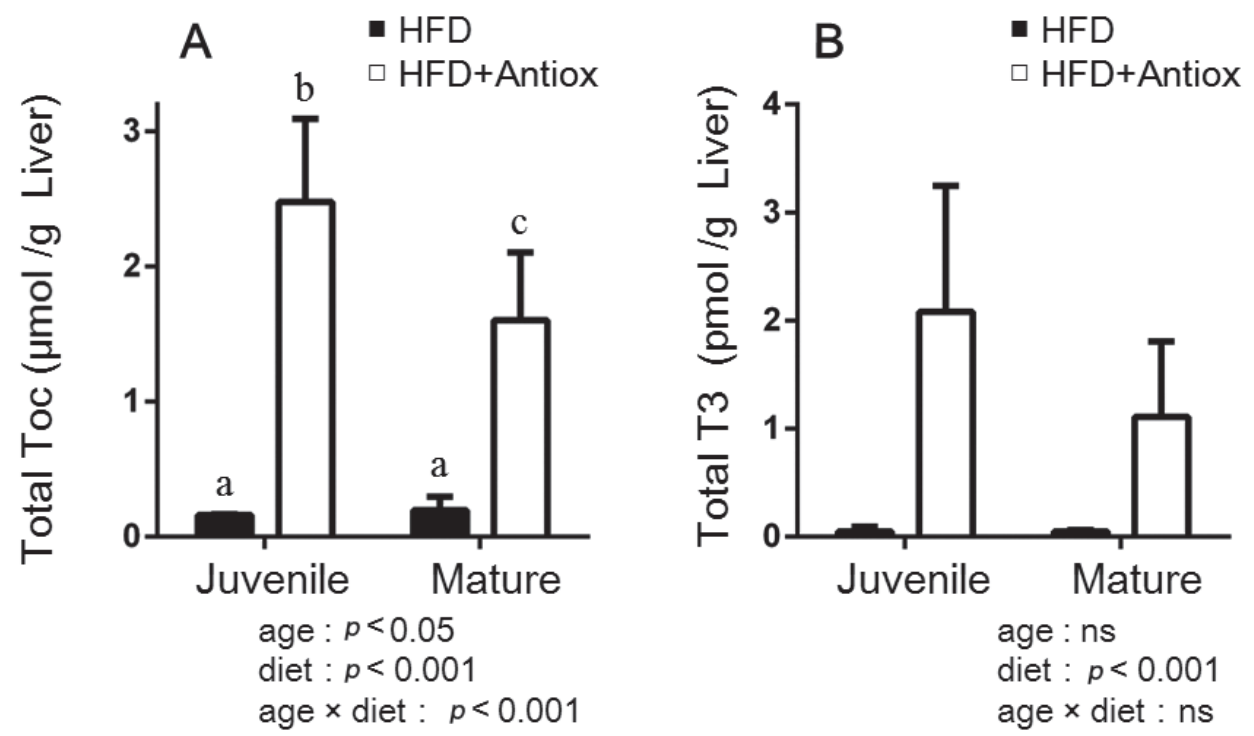

Fig. 3 Total Toc (A) and T3 (B) of liver of mature or juvenile rats fed with experimental diets for 12 weeks. Closed square; high fat diet (HFD), Open square; high fat diet supplemented with antioxidant mix (HFD+Antiox). Data are shown as means $\pm \mathrm{SD}$, Juvenile $(\mathrm{n}=8)$, Mature $(\mathrm{n}=5)$. Different letters denote statistical difference $(p<0.05)$. 


\section{S. Hayasaka, F. Kimura and S. Kato et al.}

Table 4 Liver mRNA expression levels of antioxidant-related genes in rats fed with high fat diets (HFD) or HFD supplemented with an antioxidant mix (HFD+Antiox) for 12 weeks.

\begin{tabular}{|c|c|c|c|c|c|c|c|}
\hline & \multicolumn{2}{|c|}{ Juvenile } & \multicolumn{2}{|c|}{ Mature } & \multirow{2}{*}{ age } & \multirow{2}{*}{ diet } & \multirow{2}{*}{ age $\times$ diet } \\
\hline & HFD & HFD+Antiox & HFD & HFD+Antiox & & & \\
\hline Gpxl & $1.00 \pm 0.85$ & $0.562 \pm 0.261$ & $0.690 \pm 0.330$ & $1.33 \pm 0.91$ & ns & ns & ns \\
\hline Gpx2 & $1.00 \pm 0.79^{\mathrm{a}}$ & $0.805 \pm 0.473^{\mathrm{a}}$ & $0.888 \pm 0.692^{\mathrm{a}}$ & $2.63 \pm 2.10^{\mathrm{b}}$ & $*$ & $*$ & $*$ \\
\hline Cat & $1.00 \pm 0.47$ & $0.548 \pm 0.286$ & $0.898 \pm 0.700$ & $1.12 \pm 1.05$ & $\mathrm{~ns}$ & $\mathrm{~ns}$ & $\mathrm{~ns}$ \\
\hline Mn-Sod & $1.00 \pm 0.65$ & $0.793 \pm 0.369$ & $0.906 \pm 0.441$ & $1.62 \pm 0.90$ & ns & ns & ns \\
\hline $\mathrm{Cu} / \mathrm{Zn}-\mathrm{Sod}$ & $1.00 \pm 0.86^{\mathrm{a}}$ & $1.00 \pm 0.53^{\mathrm{a}}$ & $1.06 \pm 0.38^{\mathrm{a}}$ & $3.41 \pm 2.18^{\mathrm{b}}$ & $*$ & $*$ & $*$ \\
\hline
\end{tabular}

Values are expressed as mean $\pm \mathrm{SD}$ (juvenile; $\mathrm{n}=8$, mature; $\mathrm{n}=5$ ), ${ }^{*}: p<0.05$. Different letters denote statistical difference $(p<0.05)$.

of the mature rats. Similar response was noted in juvenile rats, although to a lesser extent(Fig. 1). The commercial dietary antioxidants used in this study contained several polyphenols and n-3 unsaturated fatty acids, which are known to suppress fat accumulation ${ }^{33,34)}$. Since antioxidant supplementation decreased epididymal and perirenal-retroperitoneal fat weight as well as plasma TL, TG, T-Cho, PL and NEFA levels (Tables 2 and 3), it was speculated that the intake of antioxidants ameliorated the high-fat diet-induced body fat accumulation, thereby preventing obesityinduced phospholipid peroxidation. As an exception, liver TL, TG and T-Cho levels significantly increased upon antioxidant supplementation. The elevation of liver lipid levels has previously been reported in rats fed with high-linoleic acid diets along with vitamin $\mathrm{E}$ to an extent similar to that in this experiment ${ }^{35,36)}$. This suggests that excess intake of vitamin $\mathrm{E}$, high-fat diet, and linoleic acid may lead to the accumulation of liver lipids. Although the mechanism is still unclear, further evaluation is necessary.

Another possible factor in the reduction in $\mathrm{PCOOH}$ levels after antioxidant administration is liver vitamin E, a wellknown membrane antioxidant against phospholipid peroxidation. It was observed that liver Toc content decreased in the antioxidant treated mature rats in comparison with the antioxidant treated juvenile rats (Fig. 3), this suggests that mature rats utilized Toc to reduce oxidative stress in the liver, thus leading to the decrease in $\mathrm{PCOOH}$ levels.

Additionally, high mRNA levels of crucial antioxidant-related genes were observed in the antioxidant treated mature rats (Table 4), which may have also contributed to the decrease in $\mathrm{PCOOH}$. This finding corroborates that of a previous study where plant-derived polyphenols present in dietary antioxidants were shown to induce elevation of the gene levels of antioxidative enzymes ${ }^{37)}$. However, unvarying levels of antioxidative enzymes in the juvenile group post antioxidant treatment was contradictive. It is speculated that oxidative stress level(PCOOH levels) in juvenile group might be insufficient to elicit the upregulation of antioxidant-related genes.

Notably, we focused on $\mathrm{PCOOH}$ as the main lipid peroxi- dation marker in this study. On the basis of the above results, aging and high-fat diet feeding may also affect levels of other phospholipid hydroperoxides, including phosphatidylethanolamine hydroperoxide, as well as cholesterol hydroperoxide and triglyceride hydroperoxide. Since such other lipid peroxides are of interest, we plan to investigate them in the future.

To conclude, it was found that the increased liver $\mathrm{PCOOH}$ levels induced by the combination of aging and high-fat diet feeding were suppressed by the co-administration of a commercial antioxidant mix. Mechanistically, this antioxidant-mediated suppression may be attributed to factors such as decrease in body fat, vitamin $\mathrm{E}$ function, and/or upregulation of antioxidant-related genes. Moreover, an animal model with high tissue $\mathrm{PCOOH}$ levels can be derived from the rearing conditions described in this study. This may be a suitable model to study the relationship between phospholipid peroxidation, oxidative stressrelated diseases, and dietary antioxidants. While we focused on the effect of antioxidants on liver PCOOH levels in this study, further research is needed to validate the effectiveness of this model. Ongoing detailed analysis in our group is focused on phospholipid peroxidation in other tissues and plasma, as well as on the influence of antioxidant dosage on the levels of phospholipid peroxidation.

\section{Acknowledgements}

This study was partly supported by a JSPS KAKENHI Grant (Number 15H04497 and 15K14726).

\section{Conflict of interest}

None of the authors have any conflicts of interest. 


\section{RFERENCES}

1) Steinberg, D.; Parthasarathy, S.; Carew, T.E.; Khoo, J.C.; Witztum, J.L. Beyond cholesterol. Modifications of low-density lipoprotein that increase its atherogenicity. N. Engl. J. Med. 320, 915-924(1989).

2) Kinoshita, M.; Oikawa, S.; Hayasaka, K.; Sekikawa, A.; Nagashima, T.; Toyota, T.; Miyazawa, T. Age-related increases in plasma phosphatidylcholine hydroperoxide concentrations in control subjects and patients with hyperlipidemia. Clin. Chem. 46, 822-828(2000).

3) Kiko, T.; Nakagawa, K.; Tsuduki, T.; Suzuki, T.; Arai, H.; Miyazawa, T. Significance of lutein in red blood cells of Alzheimer's disease patients. J. Alzheimer's Dis. 28, 593-600 (2012).

4) Kiko, T.; Nakagawa, K.; Satoh, A.; Tsuduki, T.; Furukawa, K.; Arai, H.; Miyazawa, T. Amyloid beta levels in human red blood cells. PLoS One 7, e49620 (2012).

5) Nagashima, T.; Oikawa, S.; Hirayama, Y.; Tokita, Y.; Sekikawa, A.; Ishigaki, Y.; Yamada, R.; Miyazawa, T. Increase of serum phosphatidylcholine hydroperoxide dependent on glycemic control in type 2 diabetic patients. Diabetes Res. Clin. Pract. 56, 19-25 (2002).

6) Nakagawa, K.; Ninomiya, M.; Okubo, T.; Aoi, N.; Juneja, L.R.; Kim, M.; Yamanaka, K.; Miyazawa, T. Tea catechin supplementation increases antioxidant capacity and prevents phospholipid hydroperoxidation in plasma of humans. J. Agric. Food Chem. 47, 3967-3973 (1999).

7) Suzuki, K.; Nakagawa, K.; Yamamoto, T.; Miyazawa, T.; Kimura, F.; Kamei, M.; Miyazawa, T. Carbon tetrachloride-induced hepatic and renal damages in rat: inhibitory effects of cacao polyphenol. Biosci. Biotechnol. Biochem. 79, 1669-1675(2015).

8) Burdeos, G.C.; Nakagawa, K.; Kimura, F.; Miyazawa, T. Tocotrienol attenuates triglyceride accumulation in HepG2 cells and F344 rats. Lipids 47, 471-481 (2012).

9) Miyazawa, T.; Suzuki, T.; Fujimoto, K.; Kaneda, T. Phospholipid hydroperoxide accumulation in liver of rats intoxicated with carbon tetrachloride and its inhibition by dietary alpha-tocopherol. J. Biochem. 107, 689-693 (1990).

10) Miyazawa, T.; Suzuki, T.; Fujimoto, K. Age-dependent accumulation of phosphatidylcholine hydroperoxide in the brain and liver of the rat. Lipids 28, 789-793 (1993).

11） Levy, J.R.; Lesko, J.; Krieg, R.J.; Adler, R.A.; Stevens, W. Leptin responses to glucose infusions in obesity-prone rats. Am. J. Physiol. Endocrinol. Metab. 279, E10881096 (2000).

12) Lee, J.H.; Fukumoto, M.; Nishida, H.; Ikeda, I.; Sugano, M. The interrelated effects of n-6/n-3 and polyunsaturated/saturated ratios of dietary fats on the regulation of lipid metabolism in rats. J. Nutr. 119, 1893-1899 (1989).
13) Miyazawa, T.; Suzuki, T.; Fujimoto, K.; Yasuda, K. Chemiluminescent simultaneous determination of phosphatidylcholine hydroperoxide and phosphatidylethanolamine hydroperoxide in the liver and brain of the rat. J. Lipid Res. 33, 1051-1059 (1992).

14) Folch, J.; Lees, M.; Sloane Stanley, G.H. A simple method for the isolation and purification of total lipides from animal tissues. J. Biol. Chem. 226, 497-509 (1957).

15) Kato, S.; Nakagawa, K.; Suzuki, Y.; Asai, A.; Nagao, M.; Nagashima, K.; Oikawa, S.; Miyazawa, T. Liquid chromatography-tandem mass spectrometry determination of human plasma 1-palmitoyl-2-hydroperoxyoctadecadienoyl-phosphatidylcholine isomers via promotion of sodium adduct formation. Anal. Biochem. 471, 51-60 (2015).

16) Kato, S.; Nakagawa, K.; Suzuki, Y.; Suzuki, K.; Mizuochi, S.; Miyazawa, T. Preparation of 13 or 9-hydroperoxy-9Z,11E $(9 E, 11 E)$ or $10 E, 12 Z(10 E, 12 E)$-octadecadienoic phosphatidylcholine hydroperoxide. J. Oleo Sci. 63, 431-437 (2014).

17) Khoschsorur, G.; Winklhofer-Roob, B.; Rabl, H.; Auer, T.; Peng, Z.; Schaur, R. Evaluation of a sensitive HPLC method for the determination of Malondialdehyde, and application of the method to different biological materials. Chromatographia 52, 181-184(2000).

18) Burdeos, G.C.; Nakagawa, K.; Abe, T.; Kimura, F.; Miyazawa, T. Tocotrienol modulates crucial lipid metabolism-related genes in differentiated 3T3-L1 preadipocytes. Food Funct. 5, 2221-2227 (2014).

19) Bartlett, G.R. Phosphorus assay in column chromatography. J. Biol. Chem. 234, 466-468(1959).

20) Giriwono P.E.; Hashimoto, T.; Ohsaki, Y.; Shirakawa, H.; Hokazono, H.; Komai , M. Extract of fermented barley attenuates chronic alcohol induced liver damage by increasing antioxidative activities. Food Res. Int. 43, 118-124(2010).

21) Bardag-Gorce, F.; Oliva, J.; Lin, A.; Li, J.; French, B.A.; French, S.W. Proteasome inhibitor up regulates liver antioxidative enzymes in rat model of alcoholic liver disease. Exp. Mol. Pathol. 90, 123-130(2011).

22) Liu, C.M.; Zheng, Y.L.; Lu, J.; Zhang, Z.F.; Fan, S.H.; Wu, D.M.; Ma, J.Q. Quercetin protects rat liver against lead-induced oxidative stress and apoptosis. Environ. Toxico. Phar. 29, 158-166(2010).

23) Xiong, Q.; Xie, P.; Li, H.; Hao, L.; Li, G.; Qiu, T.; Liu, Y. Acute effects of microcystins exposure on the transcription of antioxidant enzyme genes in three organs (Liver, Kidney, and Testis) of male Wistar rats. J. Biochem. Mol. Toxicol. 24, 361-367(2010).

24) Djuric, Z.; Lewis, S.M.; Lu, M.H.; Mayhugh, M.; Tang, N.; Hart, R.W. Effect of varying dietary fat levels on rat growth and oxidative DNA damage. Nutr. Cancer 39, 214-219(2001). 
25) McDonald, R.B.; Carlson, K.; Day, C.; Stern, J.S.; Horwitz, B.A. Effect of gender on the response to a high fat diet in aging Fischer 344 rats. J. Nutr. 119, $1472-$ 1477 (1989).

26) Nakashima, Y.; Yokokura, A. Consumption of a high-fat diet containing lard during the growth period in rats predisposes them to favorably respond to the diet in later life. J. Nutr. Sci. Vitaminol. (Tokyo)56, 380-386 (2010).

27) Kitano-Okada, T.; Ito, A.; Koide, A.; Nakamura, Y.; Han, K.H.; Shimada, K.; Sasaki, K.; Ohba, K.; Sibayama, S.; Fukushima, M. Anti-obesity role of adzuki bean extract containing polyphenols: in vivo and in vitro effects. J. Sci. Food Agric. 92, 2644-2651 (2012).

28) Miyazawa, T. Determination of phospholipid hydroperoxides in human blood plasma by a chemiluminescence-HPLC assay. Free Radic. Biol. Med. 7, 209-217 (1989).

29) Miyazawa, T.; Yasuda, K.; Fujimoto, K.; Kaneda, T. Chemiluminescence-high performance liquid chromatography of phosphatidylcholine hydroperoxide in human blood plasma. Basic Life Sci. 49, 191-194(1988).

30) Konrad, D.; Wueest, S. The gut-adipose-liver axis in the metabolic syndrome. Physiology (Bethesda) 29, 304-313 (2014).
31) Del Rio, D.; Stewart, A.J.; Pellegrini, N. A review of recent studies on malondialdehyde as toxic molecule and biological marker of oxidative stress. Nutr. Metab. Cardiovasc. Dis. 15, 316-328(2005).

32) Janero, D.R. Malondialdehyde and thiobarbituric acidreactivity as diagnostic indices of lipid peroxidation and peroxidative tissue injury. Free Radic. Biol. Med. 9, 515-540(1990).

33) Martinez-Fernandez, L.; Laiglesia, L.M.; Huerta, A.E.; Martinez, J.A.; Moreno-Aliaga, M.J. Omega-3 fatty acids and adipose tissue function in obesity and metabolic syndrome. Prostaglandins Other Lipid Mediat. 121, 24-41(2015).

34) Meydani, M.; Hasan, S.T. Dietary polyphenols and obesity. Nutrients 2, 737-751 (2010).

35) Jack Yang, N.Y.; Desai, I.D. Effect of high levels of dietary vitamin $\mathrm{E}$ on liver and plasma lipids and fat soluble vitamins in rats. J. Nutr. 107, 1418-1426 (1977).

36) Cho, S.; Sugano, M. Effect of different levels of dietary alpha-tocopherol and linoleate on plasma and liver lipids in rats. J. Nutr. Sci. Vitaminol. (Tokyo)24, 221227 (1978).

37) Frei, B.; Higdon, J.V. Antioxidant activity of tea polyphenols in vivo: evidence from animal studies. J. Nutr. 133, 3275S-3284S (2003). 